\title{
FITOTOXICIDAD DE UN SUELO CONTAMINADO CON PETRÓLEO FRESCO SOBRE Phaseolus vulgaris L. (LEGUMINOSAE)
}

\section{Alfredo ARIAS-TRINIDAD ${ }^{1 *}$, María del Carmen RIVERA-CRUZ ${ }^{1}$ y Antonio TRUJILLO NARCÍA ${ }^{2}$}

${ }^{1}$ Colegio de Postgraduados, Campus Tabasco. Periférico Carlos A. Molina, kilómetro 3.5, carretera CárdenasHuimanguillo, Cárdenas, Tabasco, México, C. P. 86500

${ }^{2}$ Universidad Popular de la Chontalpa. Carretera Cárdenas-Huimanguillo, kilómetro 2, Ranchería Paso y Playa, Heroica Cárdenas, Tabasco, México, C. P. 86500

*Autor para correspondencia: alfredo.arias8@yahoo.com.mx

(Recibido marzo 2016; aceptado noviembre 2016)

Palabras clave: hidrocarburos, toxicidad, bioindicador, bioensayo

\section{RESUMEN}

Se evaluaron los efectos tóxicos que produce un suelo contaminado con petróleo fresco sobre plantas de Phaseolus vulgaris. Para ello se generó un índice absoluto de fitotoxicidad que integra siete parámetros, medidos a través de índices relativos de fitotoxicidad para las variables: altura, longitud radical, número de nódulos y biomasa seca (foliar, radical, del fruto y total). Asimismo, se calcularon los coeficientes de determinación $\left(\mathrm{R}^{2}\right)$. El bioensayo se realizó bajo condiciones de túnel de plástico y se evaluó los efectos tóxicos del petróleo en plantas de frijol negro (P. vulgaris L.) al cabo de 100 días. Se estableció un diseño experimental de bloques al azar en condiciones semicontroladas, con seis tratamientos con tres repeticiones por tratamiento. Las concentraciones de hidrocarburos fueron: 1500, 3000, 4500, 6000, 7500 y 9000 $\mathrm{mg} / \mathrm{kg}$ de hidrocarburos totales del petróleo (HTP) y un tratamiento testigo $(0 \mathrm{mg} / \mathrm{kg}$ ). Al día 100, el detrimento fisiológico [emergencia $\left(\mathrm{R}^{2}=0.89 ; \mathrm{b}=-3.427\right)$; altura $\left(\mathrm{R}^{2}=0.84 ; \mathrm{b}=-0.0029\right)$; longitud radical $\left(\mathrm{R}^{2}=0.62 ; \mathrm{b}=-0.001\right)$; número de nódulos $\left(\mathrm{R}^{2}=0.68 ; \mathrm{b}=-0.0092\right)$ y biomasa total $\left.\left(\mathrm{R}^{2}=0.77 ; \mathrm{b}=-0.0014\right)\right]$ de las plantas mostró una correlación negativa con la concentración de los HTP en el suelo, con diferencias entre los tratamientos (Tukey $\mathrm{p} \leq 0.05$ ). Al día 68 del ensayo se observó marchitez de las plantas a concentraciones mayores de $3000 \mathrm{mg} / \mathrm{kg} \mathrm{HTP}$.

Key words: hydrocarbons, toxicity, bioindicator, bioassay

\begin{abstract}
The toxic effects produced by soil contaminated with fresh crude oil on Phaseolus vulgaris plants was assessed. An absolute phytotoxicity index was generated, which includes seven parameters measured by a relative phytotoxicity index for the variables: height, radicular length, number of nodules, dry mass (foliar, radical, fruit and total). Likewise, the coefficients of determination $\left(\mathrm{R}^{2}\right)$ were calculated. The bioassay carried out under plastic tunnel conditions and the toxic effects of crude oil in plants $P$. vulgaris was assessed at 100 days. A randomized complete block design was used in the bioassays under semi controlled conditions, with six treatments and three
\end{abstract}


replicates at concentrations of 1500, 3000, 4500, 6000, 7500 and $9000 \mathrm{mg} / \mathrm{kg}$ of total petroleum hydrocarbons (TPH) based on dry matter and control treatment. At day 100 , the physiological damage [seedling emergence $\left(\mathrm{R}^{2}=0.89 ; \mathrm{b}=-3.427\right)$; height $\left(\mathrm{R}^{2}=0.84 ; \mathrm{b}=-0.0029\right)$; radical length $\left(\mathrm{R}^{2}=0.62, \mathrm{~b}=-0.001\right)$; nodules number $\left(\mathrm{R}^{2}=0.68 ; \mathrm{b}=-0.0092\right)$ and dry mass $\left.\left(\mathrm{R}^{2}=0.77 ; \mathrm{b}=-0.0014\right)\right]$ of the plants was inversely proportional to the TPH concentration in the soil, with differences between the treatments (Tukey $\mathrm{p} \leq 0.05$ ). At the 68 day of the assay, the withered in plants was noticed to upper concentrations of $3000 \mathrm{mg} / \mathrm{kg} \mathrm{TPH}$.

\section{INTRODUCCIÓN}

El desarrollo de la industria petrolera en el sureste de México ha generado diversos problemas ambientales. El petróleo es una mezcla de hidrocarburos parafínicos, cicloparafínicos, aromáticos y bajas cantidades de azufre, trazas de nitrógeno y compuestos de oxígeno (Seoánez et al. 1999, Ortiz-Salinas et al. 2012), además de pequeñas concentraciones de metales como V, Ni, Pb y Fe. (Figueruelo y Dávila 2001, Owamah 2013). El grado de contaminación de los suelos por petróleo depende de los componentes del ecosistema, de las propiedades físicas y químicas de los hidrocarburos del crudo y del tipo de emergencia ambiental (García-López et al. 2006, Pinkus-Rendón y Contreras-Sánchez 2012). Las principales fuentes de contaminación de los suelos por petróleo son la rotura de oleoductos, la dispersión de los derrames crónicos, los derrames de aguas residuales provenientes de los procesos de separación que se realizan en las instalaciones petroleras e, incluso, derrames de lodos de perforación y de labores de mantenimiento de los pozos petroleros (Mujica et al. 2006, Mirza et al. 2012, Riveroll-Larios et al. 2015).

Diversos estudios demuestran que los constituyentes del petróleo pueden inhibir la actividad enzimática del suelo (Alrumman et al. 2015), la germinación, el brote de meristemos, así como la disminución de la elongación radicular y la fotosíntesis en las plantas (Pérez et al. 2002, Gafari-Rahbar et al. 2012, Sangeetha y Thangadurai 2014). En México, la evaluación del impacto ambiental de un suelo contaminado con petróleo se realiza con base en parámetros geoquímicos estipulados en la NOM138-SEMARNAT/SA1-2012 (SEMARNAT 2013), la cual establece los límites máximos permisibles de hidrocarburos en el suelo y el tipo de actividad que puede realizarse en ellos: a) agrícola, forestal, pecuaria y de conservación; b) residencial y recreativa; y c) Industrial y comercial. Las normas ambientales en México no establecen el uso de parámetros biológicos para medir la toxicidad en el suelo. Asimismo, la Ley
General del Equilibrio Ecológico y Protección al Ambiente (LGEEPA) no específica el uso de plantas como bioindicadores toxicológicos (Rivera-Cruz y Trujillo-Narcía 2004). Por el contrario, a nivel internacional el Organismo para el Desarrollo y la Cooperación Económica (OECD) ha establecido la "Guía para la evaluación de sustancias químicas OECD No. 208", la cual implementa el uso de especies vegetales como biondicadores de toxicidad de sustancias químicas. La fitotoxicidad es el estudio toxicológico de un contaminante o sustancia tóxica, a partir de la respuesta fisiológica de una especie vegetal a una dosis determinada (Cruz et al. 2013). Aunque $P$. vulgaris no está considerada en la guía de la OECD No. 208 (OECD 2006) como especie fitoindicadora, autores como Beltagi (2007), Nwoko et al. (2007), Aade-Ademilua y Mbamalu (2008), Sadunishvili et al. (2009), Al-Yemeni et al. (2010) y Alba et al. (2013) han evaluado su sensibilidad en suelos contaminados con petróleo y sus derivados.

Considerando lo anterior, el objetivo de este trabajo fue evaluar el efecto tóxico de los hidrocarburos del petróleo en el crecimiento y desarrollo de plantas $P$. vulgaris, para determinar su viabilidad como especie bioindicadora de toxicidad al estimar el impacto fisiológico mediante índices de fitotoxicidad (IRF e IAF).

\section{MATERIALES Y MÉTODOS}

La colecta del suelo se realizó en las instalaciones de la Universidad Popular de la Chontalpa, ubicada en el municipio de Cárdenas, Tabasco, en las coordenadas 17057'35.1" N y 9321'58.3" W. El suelo se secó a sombra, se molió y se tamizó con malla de 0.5 $\mathrm{cm}$. El análisis físico y químico del suelo se realizó en el laboratorio de análisis del suelo, planta y agua, Colegio de Postgraduados campus Tabasco. Bajo el criterio de la NOM-021-RECNAT-2000 (SEMARNAT 2002), el suelo se identificó como: arcilloso [arcilla (51\%); limo (37\%); arena (12\%)], pH de 7.71 y contenido de materia orgánica de $1.61 \%$; estas 
variables sólo se midieron en la caracterización del suelo colectado.

Establecimiento del bioensayo. El bioensayo tuvo una duración de $100 \mathrm{~d}$ en condiciones semicontroladas de túnel de plástico, con un diseño experimental de bloques al azar, seis tratamientos con concentraciones de 1500, 3000, 4500, 6000, 7500 y $9000 \mathrm{mg} / \mathrm{kg}$ de hidrocarburos totales del petróleo (HTP) y un suelo testigo (0 mg/kg HTP). Se aplicó el Protocolo No. 208 (OECD 2006) modificado por Rivera-Cruz y Trujillo-Narcía (2004).

Los sustratos para los ensayos se prepararon a partir del suelo testigo $(0 \mathrm{mg} / \mathrm{kg}$ de HTP $)$ que se mezcló con petróleo $\left(29.5^{\circ} \mathrm{API}\right)$ disuelto en 15 $\mathrm{mL}$ de diclorometano $(99.5 \%$ de pureza marca $\mathrm{EMD}^{\mathrm{TM}}$ ) para homogeneizar el suelo; se adicionó agua destilada para una humedad entre 28 y $30 \%$, y se incubó por $24 \mathrm{~h}$ en un contenedor de vidrio de 30 x 30 x $25 \mathrm{~cm}$ de profundidad con una capacidad de $12 \mathrm{~kg}$ de suelo. Previamente se realizaron pruebas de germinación para determinar la viabilidad inicial de las semillas antes de sembrar, el resultado fue una germinación del $90 \%$ a los 5 d de la siembra (Alizaga et al. 1994).

En cada unidad experimental (UE) de cada tratamiento se sembraron cinco semillas de P. vulgaris (variedad criolla: dos mesano), en un contenedor con $10 \mathrm{~kg}$ de suelo seco; al registrar el $90 \%$ de germinación se esperó 15 días para seleccionar una de las cinco plántulas al azar y extraer las plántulas no seleccionadas. El bioensayo se mantuvo a capacidad de campo (33\% de humedad) con riegos cada tercer día y no se aportaron nutrimentos (N, P y K) para evitar interferencias en las observaciones por su presencia, lo que no permitiría conocer el efecto de los hidrocarburos del petróleo en las variables a estudiar (OECD 2006).

Medición de variables fisiológicas. Durante el bioensayo la emergencia se registró por seis días a partir de la siembra de las semillas de $P$. vulgaris en las UE. La medición de la altura (AL) se realizó a los $12,25,39,54,68,82$ y 96 días después de la siembra (dds). La longitud radical (LR), número de nódulos (NN) y biomasa seca aérea (BSA), radical (BSR) y total (BST) se midieron al término del bioensayo (100 dds) al hacerse el muestreo destructivo (cosecha) de las UE de los tratamientos. Se contabilizó el NN de la raíz de cada planta y la LR se midió con una regla en cm, desde la base del tallo al extremo más largo de la raíz de cada planta. Para determinar la biomasa seca se cortó y separó la parte aérea (frutos, tallos y hojas) y radical (raíz y nódulos) de cada planta y se colocaron en bolsas de papel etiquetadas, poste- riormente se secaron en una estufa $\left(75^{\circ} \mathrm{C}, 48 \mathrm{~h}\right)$, el material seco se pesó en una balanza analítica para obtener los valores de BSA, BSR y BSF.

Índices de fitotoxicidad. El índice relativo de fitotoxicidad (IRF) y el índice absoluto de fitotoxicidad (IAF) de $P$. vulgaris se determinaron con el método de Vázquez-Luna et al. (2010) modificado de Rivera-Cruz y Trujillo-Narcía (2004). El IRF indica el deterioro de las variables fisiológicas de $P$. vulgaris a causa del contenido HTP de cada tratamiento. El IAF indica el efecto fitotóxico total de $P$. vulgaris por tratamiento, considerando los IRF de todas las variables fisiológicas. Para ello se aplicaron las siguientes ecuaciones:

$\operatorname{IRF}(x)=1-\left[\frac{T p R 1}{T t}\right]$
$I A F=\frac{\sum_{i=1}^{n} I R F}{T t} \quad i=1,2 \cdots t$

Donde:

$\operatorname{IRF}(x)=$ índice relativo de fitotoxicidad.

$I A F=$ índice absoluto de fitotoxicidad.

$T p=$ tratamiento con HTP.

$T t=$ tratamiento testigo.

$R 1$ : repetición $1 \ldots n$.

$n=$ número de variables.

Los resultados se interpretaron bajo el criterio de: si $I R F_{(x)}$, es igual a cero: el efecto es neutro (no afecta a la variable), pero si es mayor de cero el efecto es negativo (inhibición); si el $I R F_{(x)}$, es menor de cero el efecto es positivo sobre la variable (estimulación).

Se utilizó el programa SPSS para determinar el análisis de varianza y los coeficientes de determinación $\left(\mathrm{R}^{2}\right)$ de cada una de las variables fisiológicas de P. vulgaris $(\mathrm{p} \leq 0.05)$.

\section{RESULTADOS}

Las variables presentaron diferencias estadísticas significativas al aumentar la concentración de HTP en el suelo. La emergencia mostró una inhibición significativa en los tratamientos con hidrocarburos del petróleo $\left(\mathrm{R}^{2}=0.8907 ; \mathrm{b}=-3.427\right)$ (Fig. 1.). En suelos con 1500 y $3000 \mathrm{mg} / \mathrm{kg}$ de HTP esta variable registró una inhibición del $20 \%$ y $32 \%$ respectivamente, en relación con el tratamiento testigo, mientras que a concentraciones de $9000 \mathrm{mg} / \mathrm{kg}$ de HTP se inhibió un $80 \%$ con respecto al testigo. A los 68 dds las plantas de P. vulgaris en suelos a 

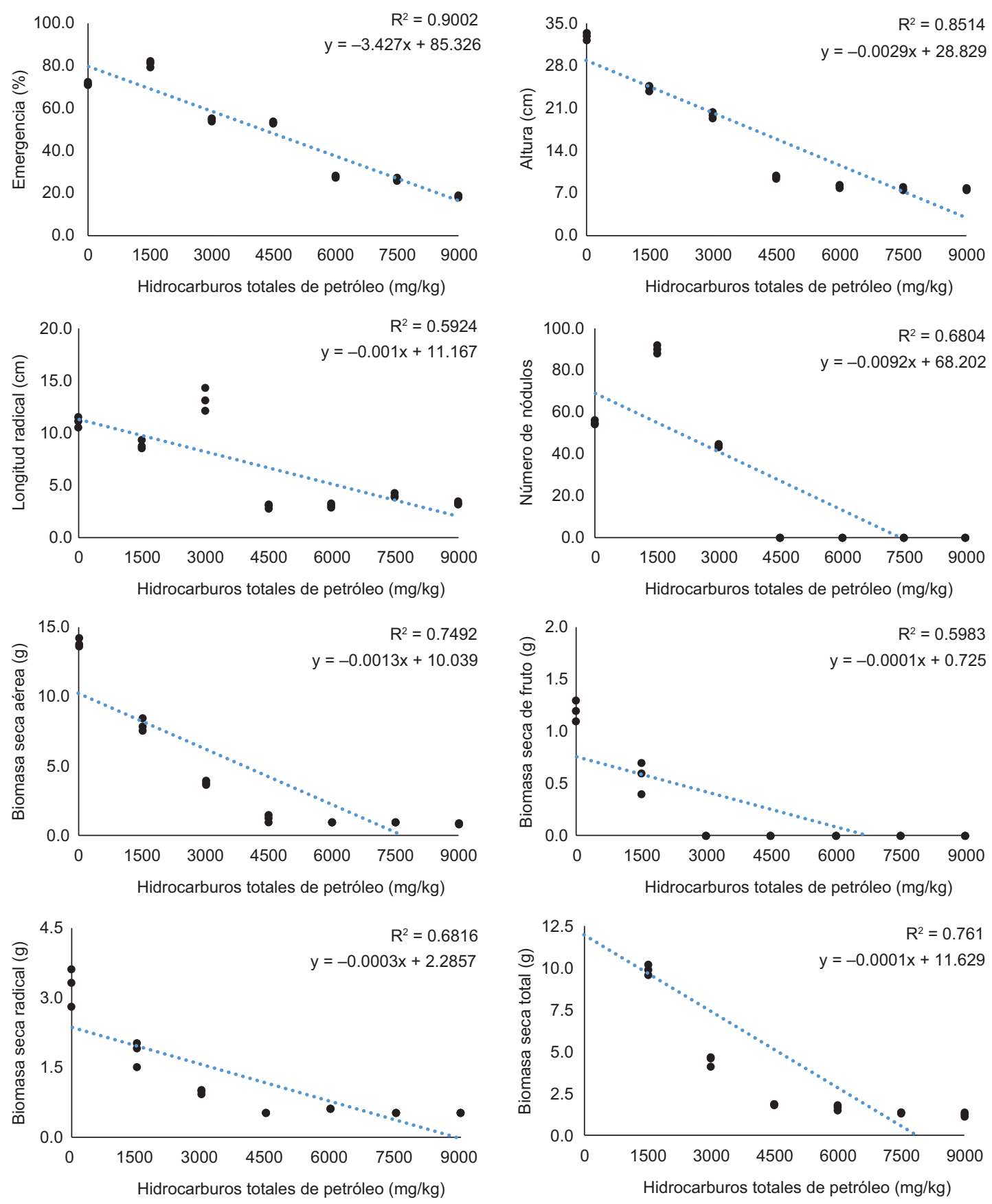

Fig. 1. Regresión lineal de las variables fisiológicas de Phaseolus vulgaris (emergencia, altura, longitud radical, número de nódulos, biomasa seca aérea, biomasa seca de frutos, biomasa seca radical y biomasa seca total) en relación al contenido de hidrocarburos del petróleo $(\mathrm{p} \leq 0.05)$.

concentraciones de 4500, 6000, 7500 y $9000 \mathrm{mg} / \mathrm{kg}$ de HTP se marchitaron (Cuadro I). Sólo las plantas que crecieron en suelos con 1500 y $3000 \mathrm{mg} / \mathrm{kg}$ de HTP soportaron la exposición al petróleo durante todo el experimento (Fig. 1).
Los resultados de NN $\left(\mathrm{R}^{2}=0.68 ; \mathrm{b}=-0.0092\right)$ y $\mathrm{LR}$ $\left(R^{2}=0.62 ; b=-0.001\right)$ presentaron variaciones significativas entre los tratamientos (Fig. 1; Cuadro I). El NN en suelos con $1500 \mathrm{mg} / \mathrm{kg}$ de HTP fue mayor en un $60 \%$, mientras que la LR se redujo en un 
CUADRO I. VALORES DE ÍNDICES RELATIVOS Y ABSOLUTOS DE FITOTOXICIDAD EN PLANTA DE Phaseolus vulgaris DE SUELOS CONTAMINADOS CON PETRÓLEO (100 DÍAS)

\begin{tabular}{lccccccc}
\hline \multirow{2}{*}{$\begin{array}{l}\text { Tratamiento } \\
\text { (mg/kg de HTP) }\end{array}$} & Altura & $\begin{array}{c}\text { Longitud } \\
\text { radical }\end{array}$ & $\begin{array}{c}\text { Biomasa } \\
\text { seca área }\end{array}$ & $\begin{array}{c}\text { Biomasa } \\
\text { seca de fruto }\end{array}$ & $\begin{array}{c}\text { Biomasa } \\
\text { seca radical }\end{array}$ & $\begin{array}{c}\text { Número de } \\
\text { nódulos }\end{array}$ & $\begin{array}{c}\text { Índice absoluto } \\
\text { de fitotoxicidad }\end{array}$ \\
\cline { 2 - 6 } Testigo (0) & 0 & 0 & 0 & 0 & 0 & 0 & 0 \\
1500 & 0.29 & 0.28 & 0.45 & 0.48 & 0.36 & -0.59 & 0.26 \\
3000 & 0.42 & 0.30 & 0.73 & 1 & 0.69 & 0.20 & 0.58 \\
4500 & 0.71 & 0.73 & 0.91 & 1 & 0.88 & 1 & 0.87 \\
6000 & 0.76 & 0.76 & 0.92 & 1 & 0.89 & 1 & 0.88 \\
7500 & 0.76 & 0.74 & 0.93 & 1 & 0.91 & 1 & 0.89 \\
9000 & 0.77 & 0.76 & 0.93 & 1 & 0.91 & 1 & 0.90 \\
\hline
\end{tabular}

$26 \%$ respecto al testigo. La BS registró diferencias estadísticas entre los tratamientos. En los tratamientos a concentraciones de 1500 y $3000 \mathrm{mg} / \mathrm{kg}$ de HTP la $\mathrm{BST}\left(\mathrm{R}^{2}=0.77 ; \mathrm{b}=-0.0014\right)$ disminuyó en un $36 \mathrm{y}$ $69 \%$, respectivamente, con respecto al testigo. Para el caso de la BSF $\left(\mathrm{R}^{2}=0.63\right)$, el único el tratamiento en el que se produjeron vainas fue el de $1500 \mathrm{mg} / \mathrm{kg}$ de HTP, sin embargo, el valor fue un $54 \%$ menor que el del testigo.

Los IRF muestran una relación directamente proporcional entre el contenido de HTP y las variables de $P$. vulgaris estudiadas. La BSA y BSR fueron las más afectadas por la concentración de HTP en los suelos. Sin embargo, el NN a $1500 \mathrm{mg} / \mathrm{kg}$ HTP presentó una estimulación del $59 \%$ respecto al testigo. Los IAF en los suelos con 1500 y $3000 \mathrm{mg} / \mathrm{kg}$ de HTP registraron una reducción del 26 y $58 \%$ respectivamente, en relación con el testigo, lo que demuestra el daño provocado a $P$. vulgaris por los hidrocarburos del petróleo, a pesar de su desarrollo vegetal (Cuadro I).

\section{DISCUSIÓN}

El severo daño fisiológico de $\mathrm{P}$. vulgaris durante el experimento, se atribuye a las condiciones adversas que se generan por los cambios físicos y químicos del suelo por los hidrocarburos (aumento del carbono orgánico, concentración de nitrógeno, $\mathrm{pH}$, conductividad eléctrica y contenido de arcilla). Así como por los metales pesados (cadmio, cobre, hierro, zinc y plomo) presentes en el petróleo (Khan et al. 2013). Se ha demostrado que la fracción soluble en agua y los lixiviados de los hidrocarburos del petróleo, reducen el metabolismo y desarrollo fisiológico de las plantas tras un derrame, al inhibir la madurez fisiológica de las raíces y propiciar la reducción en la biomasa foliar (Blankenship y Larson 1978, Sadunishvili et al. 2009, Hawrot-Paw y Bąkowska 2014). Este efecto deriva como respuesta de la penetración de los hidrocarburos a nivel celular, los cuales inducen cambios metabólicos intracelulares que conducen a la marchitez (Abha y Swaranjit-Singh 2012). Asimismo, pueden perjudicar el crecimiento del embrión por la inhibición de la comunidad microbiana tanto del suelo como de la rizósfera (Sangeetha y Thangadurai 2014), y por el efecto hidrofóbico que restringe el contenido de agua y oxígeno (Akaninwor et al. 2007, Ferreira do Nascimento et al. 2013).

En cuanto a la reducción de la AL, BS y LR por efecto de los HTP, Hernández-Acosta et al. (2006), Adams et al. (2008) y Al-Yemeni et al. (2010) indican que el petróleo puede afectar la fotosíntesis y el contenido de clorofila en la vegetación, lo que podría generar una baja producción de biomasa. La presencia de HTP puede inhibir el crecimiento foliar de las plantas para aprovechar la mayor cantidad de sus recursos y energía para consolidar su fracción radical (Banks et al. 2003, Kechavarzi et al. 2007, Bona et al. 2011). Asimismo, Syafruddin y Efendi (2014) concluyeron que un factor importante en la producción de biomasa seca en especies fitorremediadora de suelos contaminados con petróleo, es la densidad y el tipo de poblaciones microbianas de la rizósfera. Por otra parte, estudios en gramíneas y leguminosas explican que las condiciones de anegamiento permiten el aumento de la materia vegetal por el transporte de material fotosintético a las raíces, esto favorece el crecimiento y desarrollo del tejido fotosintético. Sin embargo, esta producción de materia vegetal puede variar dependiendo de los exudados radicales, el tipo de sistema radical y la fase fisiológica de la especie vegetal (Huang et al. 2004, Liu et al. 2011, Ciotti et al. 2014).

A pesar de la estimulación (-59, IRF) de la producción de nódulos de $P$. vulgaris en el tratamiento a $1500 \mathrm{mg} / \mathrm{kg}$ de HTP, se asume que este efecto es una forma de la planta de contrarrestar las condiciones 
adversas de los HTP para mejorar su crecimiento y desarrollo fisiológico (Adam-Duncan 2002, Kechavarzi et al. 2007). Ciertos estudios indican que la producción de nódulos se debe a la presencia de ácidos y compuestos biosintéticos aromáticos que estimulan la presencia de bacterias del genero Rhizobium; las cuales promueven el crecimiento de las plantas; al invadir las raíces de las leguminosas y forman nódulos para fijar nitrógeno atmosférico como amoníaco (Parke et al. 1985, Teng et al. 2015). Dorn y Salanitro (2000) y Baek et al. (2004) explican que bajos contenidos de hidrocarburos del petróleo puede estimular el desarrollo y crecimiento de las especies vegetales, e incluso los efectos no son observables a concentraciones inferiores a $1000 \mathrm{mg} / \mathrm{kg}$ de HTP. Asimismo, Odokuma y Ubogu (2014) registraron un incremento del $22 \%$ respecto al testigo en la materia seca total de suelos contaminados con petróleo a 1 $\%$ p/p. Por el contrario, Méndez-Natera et al. (2005) y Nwoko et al. (2007) reportaron una disminución en la formación de nódulos en las leguminosas Vigna unguiculata y $P$. vulgaris, respectivamente, directamente proporcional al contenido de HTP. Asimismo, Ekpo y Nkanang (2010), explican que los derivados del petróleo, como el diésel, afectan el desarrollo de nódulos y la multiplicación de bacteroides dentro de los nódulos de las leguminosas. Spiares y Kenworthy (2001) señalan que la inhibición de nódulos en algunas leguminosas se debe al bajo contenido de agua y oxígeno, la pérdida de nutrimentos, el descenso de la actividad microbiana y el incremento de metales pesados que el petróleo causa en los suelos.

Los índices fitotóxicos generados en esta investigación son similares a los reportados por VázquezLuna et al. (2010), que demuestran de forma cuantitativa los daños causados en el desarrollo de plantas de $P$. vulgaris en presencia de petróleo en el suelo. Resultados similares se registraron en los estudios de Varnero et al. (2007) quienes identificaron, a partir de índices toxicológicos, que la germinación es la variable más sensible (efecto fitotóxico) a sustratos orgánicos en composta (café instantáneo, fruta seca, residuos vitivinícolas y guano de aves). Camarillo-Ravelo et al. (2015) reportaron la aplicación de índices de germinación y radicular para asignar los valores NOEL (concentración a la que los efectos no son observables) y LOEL (concentración mínima a la que los efectos son observables) de cuatro especies vegetales (Lactuca sativa, Cucumis sativus, Brassica juncea y Nasturtium officinale) en jales mineros y observaron que el índice radical es la alternativa más viable para evaluaciones de fitotoxicidad. Trujillo-Narcía et al. (2014) concluyeron que el cálculo de índices de impacto ecotoxicológico permite identificar parámetros sensibles para evaluar la calidad de la restauración de fluvisoles contaminados con petróleo crudo.

\section{CONCLUSIONES}

El estrés vegetal de P. vulgaris en suelos contaminados con HTP fue directamente proporcional a la concentración del tratamiento, las variables de emergencia, como altura y biomasa total, fueron los parámetros más sensibles. Los resultados del estudio demuestran la sensibilidad de $P$. vulgaris como una especie bioindicadora para el monitoreo de un proceso de remediación y el impacto sobre un ecosistema de un pasivo o emergencia ambiental. Los índices de fitotoxicidad (IRF y IAF) permiten estimar y relacionar en forma porcentual el daño fisiológico de las plantas en bioensayos toxicológicos de suelos contaminados con hidrocarburos del petróleo fresco.

\section{AGRADECIMIENTOS}

Al Colegio de Postgraduados, Campus Tabasco y a la Universidad Popular de la Chontalpa, por el apoyo recibido durante la investigación.

\section{REFERENCIAS}

Aade-Ademilua O.E. y Mbamalu J. (2008). The growth and development of Phaseolus vulgaris L. under petrol and diesel-oil polluted water irrigation. Res. J. Environ. Toxicol. 2 (2), 27-34. DOI: 10.3923/rjet.2008.27.34

Abha S. y Swaranjit-Singh C. (2012). Hydrocarbon pollution: effects on living organisms, remediation of contaminated, environments, and effects of heavy metals co-contamination on bioremediation. En: Introduction to enhanced oil recovery (EOR) processes and bioremediation of oil-contaminated sites (L. Romero-Zerón, Ed.). InTech Europe, Rijeka, Croatia, pp. 185-206. DOI: $10.5772 / 48014$

Adam G. y Duncan H. (2002). Influence of diesel fuel on seed germination. Environ. Pollut. 120 (2), 363-370. DOI: $10.1016 / \mathrm{S} 0269-7491(02) 00119-7$

Adams R.H., Zavala C.J. y Morales G.F. (2008). Concentración residual de hidrocarburos en suelo del trópico, II: Afectación a la fertilidad y su recuperación. Interciencia 33 (7), 483-489. 
Akaninwor J.O., Ayeleso A.O. y Monago C.C. (2007). Effect of different concentrations of crude oil (Bonny light) on major food reserves in guinea corn during germination and growth. Sci. Res. Essays 2 (4), 127-131.

Alba G.I., Cuevas M.C. y Bustos E. (2013). Comparing the electroremediation of Gleysol soil contaminated with hydrocarbons with triton X-114 washing and bioremediation with solid cultures employing agroindustrial residues. Int. J. Electrochem. Sci. 8 (1), 6365-6376.

Alizaga R., Mello V.D.C., dos Santos D.S.B. e Irigon, D.L. (1994). Evaluación del vigor en semilla de Phaseolus vulgaris y su relación con la emergencia en el campo. Rev. Agron. Costarricense 18 (2), 227-234.

Alrumman S., Standing B.D. y Paton I.G. (2015). Effects of hydrocarbon contamination on soil microbial community and enzyme activity. J. King Saud Univ. Sci. 27 (1), 31-41. DOI: 10.1016/j.jksus.2014.10.001

Al-Yemeni M.N., Siddiqui M.H. y Wijaya L.F. (2010). Effect of petroleum polluted soil on the performance of Phaseolus vulgaris L. American-Eurasian. J. Agric. Environ. Sci. 7 (4), 427-432.

Baek K.H., Kim H.S., Oh H.M., Yoon B.D., Kim J. y Lee I.S. (2004). Effects of crude oil, oil components, and bioremediation on plant growth. J. Environ. Sci. Health A 39 (9), 2465-2472. DOI: 10.1081/LESA-200026309

Banks M.K., Schwab P., Liu B., Kulakow P.A., Smith J.S. y Kim R. (2003). The effect of plants on the degradation and toxicity of petroleum contaminants in soil: a field assessment. Adv. Biochem. Eng. Biot. 78, 76-96. DOI: $10.1007 / 3-540-45991-X \_3$

Beltagi M.S. (2007). Phytotoxicity of methyl tert-butyl ether (MTBE) to common bean (Phaseolus vulgaris L.) Plants. Pak. J. Biol. Sci. 10 (21), 3847-3852. DOI: $10.3923 /$ pjbs.2007.3847.3852

Blankenship D.W. y Larson R.A. (1978). Plant growth inhibition by the water extract of a crude oil. Water, Air, Soil Poll. 10 (4), 471-476. DOI: 10.1007/BF00250012

Bona C., Rezende M.I., Oliveira S.G. y Souza A.L. (2011). Effect of soil contaminated by diesel oil on the germination of seeds and the growth of Schinus terebinthifolius Raddi (Anacardiaceae) seedlings. Braz. Arch. Biol. Tehn. 54 (6), 1379-1387. DOI: $10.1590 / \mathrm{S} 1516-89132011000600025$

Camarillo-Ravelo D., Barajas-Aceves M. y RodríguezVázquez R. (2015) Evaluación de la fitotoxicidad de jales mineros en cuatro especies empleadas como bioindicadores de metales pesados. Rev. Int. Contam. Ambie. 31 (2), 133-143.

DOI: 10.1016/j.egypro.2011.03.149

Ciotti E.M., Castelán M.E., Hack C.M., Porta M. y González A. M. (2014). Tolerancia de leguminosas herbáceas estivales a condiciones de anegamiento temporal. Tropical Grasslands 2 (1), 278-286.

DOI: 10.17138/TGFT(2)278-286

Cruz J.M., Lopes P.R.M., Montagnolli R.N., Tamada I.S., Silva N.M.M.G. y Bidoia E.D. (2013). Phytotoxicity of soil contaminated with petroleum derivatives and biodiesel. Ecotoxicol. Environ. Saf. 8 (1), 49-54.

DOI: 10.5132/eec.2013.01.007

Dorn P.B. y Salanitro J.P. (2000). Temporal ecological assessment of oil contaminated soils before and after bioremediation. Chemosphere 40 (4), 419-426.

DOI: $10.1016 / \mathrm{S} 0045-6535(99) 00304-5$

Ekpo M.A. y Nkanang A.J. (2010). Nitrogen fixing capacity of legumes and their Rhizospheral microflora in diesel oil polluted soil in the tropics. J. Petroleum Gas Eng. 1 (4), 76-83.

Ferreira do Nascimento T.C., Santos-Oliveira F.J. y Pessoa de Franca F. (2013). Biorremediación de un suelo tropical contaminado con residuos aceitosos intemperizados. Rev. Int. Contam. Ambie. 29, 21-28.

Figueruelo E.J. y Dávila M.M. (2001). Química física del medio ambiente. Ed. Reverté, México D.F., México, 619 pp.

Gafari-Rahbar F., Kiarostami K. y Shirdam R. (2012). Effects of petroleum hydrocarbons on growth, photosynthetic pigments and carbohydrate levels of sunflower. J. Food Agric. Environ. 10 (1), 773-776.

García-López E., Zavala-Cruz J. y Palma-López D.J. (2006). Caracterización de las comunidades vegetales en un área afectada por derrames de hidrocarburos. Terra Latinoamericana 24 (1), 17-26.

Hawrot-Paw M. y Bąkowska T. (2014). Growth and development of selected plant species in the phytoremediation of diesel oil contaminated soil. Environ. Prot. Eng. 40 (4), 5-13. DOI: 10.5277/epe140401

Hernández-Acosta E., Gutiérrez-Castorena M.C., Rubiños-Panta J.E. y Alvarado-López J. (2006). Caracterización del suelo y plantas de un sitio contaminado con hidrocarburos. Terra Latinoamericana 24 (4), 463-470.

Huang X.D., El-Alawi Y., Penrose D.M., Glick B.R. y Greenberg B.M. (2004). Responses of three grass species to creosote during phytoremediation. Environ. Pollut. 130 (3), 453-463.

DOI: 10.1016/j.envpol.2003.12.018

Kechavarzi C., Pettersson K., Leeds-Harrison P., Ritche L. y Ledin, S. (2007). Root establishment of perennial ryegrass ( $L$. perenne) in diesel contaminated subsurface soil layers. Environ. Pollut. 145 (1), 68-74.

DOI: $10.1016 /$ j.envpol.2006.03.039

Khan S.R., Kumar N.J.I., Kumar N.R. y Patel G.J. (2013). An assessment of physicochemical properties, heavy metal content and fungal characterization of an old gasoline-contaminated soil site Anand, Gujarat, India. Int. J. Environ. 2 (1), 137-143.

DOI: 10.3126/ije.v2i1.9219 
Liu J., Duan C., Zhang X., Zhu Y. y Xiaoyan L. (2011). Potential of Leersia hexandra Swartz for phytoextraction of Cr from soil. J. Hazard. Mater. 188 (1-3), 85-91. DOI: 10.1016/j.jhazmat.2011.01.066

Méndez-Natera J.R., Mújica B.C.F. y Pino M.F.B. (2005). Efecto de la contaminación con petróleo sobre los caracteres de la nodulación en el cultivo de frijol ( $\mathrm{Vi}$ gna unguiculata (L.) Walp.) en dos suelos del estado Monagas. Revista UDO Agrícola 5 (1), 81-87.

Mirza R., Faghiri I. y Abedi E. (2012). Contamination of polycyclic aromatic hidrocarbons in surface sediments of khure-musa estuarine, Persian Gulf. World J. Fish Marine Sci. 4 (2), 136-141.

DOI: 10.5829/idosi.wjfms.2012.04.02.56332

Mújica B.C.F., Méndez N.J.R. y Pino M.F.B. (2006). Crecimiento de plántulas de frijol (Vigna unguiculata (L.) Walp.) en dos suelos contaminados con petróleo. Rev. Tecnológica ESPOL. 19 (1), 17-24.

Nwoko C.O., Okeke P.N., Agwu O.O. y Akpan I.E. (2007). Performance of Phaseolus vulgaris L. in a soil contaminated with spent-engine oil. Afr. J. Biotechnol. 6 (16), 1922-1925.

Odokuma L.O. y Ubogu M. (2014). Phragmitis australies growth and tolerance to crude oil contamination in Mangrove swamp soil. J. Bioremed. Biodeg. 5, 256261. DOI: $10.4172 / 2155-6199.1000256$

OECD (2006). Test No. 208: Terrestrial plant test: seedling emergence and seedling growth test. Guía para la evaluación de sustancias químicas. Organization for Economic Cooperation and Development, 21 pp. DOI: 10.1787/9789264070066-en

Ortiz-Salinas R., Cram S. y Sommer I. (2012). Hidrocarburos aromáticos policíclicos (HAPs) en suelos de la llanura aluvial baja del estado de Tabasco, México. Revista Universidad y Ciencia 28 (2), 131-144.

Owamah H.I. (2013). Heavy metals determination and assessment in a petroleum impacted driver in the niger delta región of Nigeria. J. Pet. Environ. Biotechnol. 4, 135-138. DOI: $10.4172 / 2157-7463.1000135$

Parke D., Rivelli M. y Ornston L.N. (1985). Chemotaxis to aromatic and hydroaromatic acids:comparison of Bradyrhizobium japonicum and Rhizobium trifolii. J. Bacteriol. 163 (2), 417-422.

Pérez V.J., García E.G. y Esparza G.F. (2002). Papel ecológico de la flora rizosférica en fitorremediación. Avance y Perspectiva 21 (1), 297-300.

Pinkus-Rendón M.J. y Contreras-Sáncez A. (2012). Impacto socioambiental de la industria petrolera en Tabasco: el caso de la Chontalapa. Revista LiminaR Estudios Sociales y Humanisticos 10 (2), 122-144.

Rivera-Cruz M.C. y Trujillo-Narcía A. (2004). Estudio de toxicidad vegetal en suelos con petróleos nuevo e intemperizado. Interciencia 29 (7), 369-376.
Riveroll-Larios J., Escalante-Espinosa E., Fócil-Monterrubio R.L. y Díaz-Ramírez I.J. (2015). Biological activity asessment in mexican tropical soil with different hydrocarbon contamination histories. Water, Air, Soil Poll. 226 (10), 353-362.

DOI: $10.1007 / \mathrm{s} 11270-015-2621-1$

Sadunishvili T., Kvesitadze E., Betsiashvili M., Kuprava N., Zaalishvili G. y Kvesitadze G. (2009). Influence of hydrocarbons on plant cell ultrastructure and main metabolic enzymes. World Academy of Science, Engineering and Technology 3 (9), 429-434.

Sangeetha J. y Thangadurai D. (2014). Effect of biologically treated petroleum sludge on seed germination and seedling growth of Vigna unguiculata (L.) Walp. (Fabaceae). Braz. Arch. Biol. Tehn. 57 (3), 427-433. DOI: $10.1590 / \mathrm{S} 1516-89132014005000011$

SEMARNAT (2002). Proyecto de Norma Oficial Mexicana NOM-021-RECNAT-2000. Especificaciones de fertilidad, salinidad y clasificación de suelos, estudio, muestreo y análisis. Secretaría de Medio Ambiente y Recursos Naturales. Diario Oficial de la Federación. 31 de diciembre de 2002.

SEMARNAT (2013). Norma Oficial Mexicana NOM138-SEMARNAT/SAI-2012. Límites máximos permisibles de hidrocarburos en suelos y lineamientos para el muestreo y la remediación. Secretaría de Medio Ambiente y Recursos Naturales. Diario Oficial de la Federación. 10 de septiembre de 2013.

Seoánez C.M., Chacón A.J., Gutiérrez A. y Angulo I. (1999). Contaminación del suelo: estudios, tratamientos y gestión. Mundi-Prensa, Barcelona, España, $352 \mathrm{pp}$.

Spiares J.D. y Kenworthy K.E. (2001). Root and shoot biomass of plant seeded in crude oil contaminated soil. Texas J. Agric. Nat. Resources 14 (1), 117-124.

Syafruddin S. y Efendi R. (2014). Effect of plant species and bacterial isolates to development of the plant biomass and total microorganisms of contaminated soil. Int. J. Agric. Res. 9 (1), 17-28. DOI: 10.3923/ ijar.2014.17.28

Teng Y., Wang X., Li L., Li Z. y Luo Y. (2015). Rhizobia and their bio-partners as novel drivers for functional remediation in contaminated soils. Front. Plant Sci. 6 (32), 1-11. DOI: 10.3389/fpls.2015.00032

Trujillo-Narcía A., Rivera-Cruz M. C., Lagunes-Espinoza L. C., Palma-López D. J., Sánchez-Soto S. y RamírezValverde G. (2014) Biological parameters of the restoration of soil polluted by crude oil. Ecosistemas y recursos agropecuarios 1 (2), 107-122.

Varnero M.M.T., Rojas A.C. y Orellana R.R. (2007). Índices de fitotoxicidad en residuos orgánicos durante el compostaje. Rev. Cienc. Suelo Nutr. 7 (1), 28-37. DOI: $10.4067 / \mathrm{S} 0718-27912007000100003$ 
Vázquez-Luna D., Castelán-Estrada M., Rivera-Cruz M.C., Ortiz-Ceballos A.I. y Izquierdo R.F. (2010). Crotalaria incana L. y Leucaena leucocephala Lam.
(Leguminosae): especies indicadoras de toxicidad por hidrocarburos de petróleo en el suelo. Rev. Int. Contam. Ambie. 26 (3), 183-191. 